\title{
Current Short-Range Tests of the Gravitational Inverse Square Law
}

\author{
Joshua C. Long \\ Los Alamos Neutron Science Center, \\ LANSCE-3, MS-H855, Los Alamos NM 87545 \\ John C. Price \\ Department of Physics, University of Colorado, Boulder CO 80309
}

(Dated: October 26, 2018)

\begin{abstract}
Motivated in large part by the possibility of observing signatures of compact extra dimensions, experimental searches for deviations from Newtonian gravity at short distances have improved in sensitivity by many orders of magnitude in the past five years. We review the essential features of the experiments responsible for the current limits on new effects in the range from a few microns to a few centimeters, and discuss prospects for the near future.
\end{abstract}




\section{INTRODUCTION}

There are several motivations for the experimental study of the gravitational force at short distances. Most generally, gravity is poorly understood below one centimeter. Up until the year 2000 it was not known if gravity obeyed the inverse square law below distance scales as large as several millimeters. Second (and closely related) is the opportunity to explore a large parameter space for new forces in nature. Even with the recent advances in short-distance measurements, experimental limits allow for new forces at least a million times stronger than gravity acting over distances resolvable to the unaided eye. Third, due to developments in theoretical particle physics, short-range gravity experiments have become laboratories to test for specific new physics beyond the Standard Model. Many recent models attempting to unify gravity and the other fundamental forces in the same theoretical framework predict modifications of gravity in the range of about a millimeter. Among these, predicted signatures of extra spatial dimensions have been most responsible for stimulating new experiments.

In these experiments, new phenomena, including the influence of extra dimensions, are expected to manifest themselves as departures from the Newtonian inverse square law. A common way to parameterize these effects is with the Yukawa interaction. The potential energy due to gravity and an additional Yukawa force between test masses $m_{1}$ and $m_{2}$ is given by:

$$
V=-\frac{G m_{1} m_{2}}{r_{12}}\left[1+\alpha \exp \left(-r_{12} / \lambda\right)\right]
$$

where $G$ is the Newtonian gravitational constant, $r_{12}$ is the distance between the test masses, $\alpha$ is the strength of the new interaction relative to gravity, and $\lambda$ is the range. The Yukawa potential arises when the interaction between test masses $m_{1}$ and $m_{2}$ in the above expression is mediated by a massive field. In the case of a massless mediator with infinite range, such as the graviton, the usual $1 / r$ potential is recovered. For cases in which the mediator couples to some quantity other than mass (for example, baryon number), $\alpha$ can acquire a dependence on the composition of the test masses. This effect is expected to be at most a small fraction of $\alpha$. It is addressed in experimental tests of the equivalence principle, which are conducted over ranges in which gravity is relatively well-measured.

This review concentrates on the search for new effects in the interaction range between a few microns and a few centimeters. As the experiments in this range are at or near 
gravitational sensitivity, it is here where phenomena associated with the extradimensional models of greatest interest are most likely to appear in the near future. There are also other specific predictions of new physics in this regime, some indirectly related to the physics of extra dimensions, which may soon be observable. Experimental programs motivated in part by the physics of extra dimensions have begun to explore other distance ranges as well. A significant effort is active in the range below $100 \mathrm{~nm}$ in connection with recent Casimir force measurements, as reviewed in Refs. [1] and [2].

To date, no convincing signal of a departure from Newtonian gravity has been observed. In the absence of signals, experiments commonly report results in terms of limits on the strength parameter $\alpha$ for a given range $\lambda$. The current experimental limits are shown in Fig. 1, together with recent theoretical predictions.

\section{THEORETICAL MOTIVATIONS}

Several predictions of new physics which can be tested in current short-range experiments arise in models addressing the unification of the fundamental forces. The leading candidate models are inspired by string or M-theory, which has to be formulated in more than three spatial dimensions. The extra dimensions are typically compactified at a scale on the order of the Planck length $\left(L_{P} \sim 10^{-35} \mathrm{~m}\right.$ or $\left.L_{P}^{-1}=M_{P} \sim 10^{19} \mathrm{GeV}\right)$, the presumed gravitational unification scale, or concealed from direct detection by other mechanisms. Recently several models have been discovered which hinge on at least one of the extra dimensions remaining larger than $L_{P}$, with significant consequences for short-range experiments. String theories also contain scalar fields called moduli, which parameterize the size and shape of the extra dimensions and can mediate new short-range forces. Theoretical models are treated extensively in other parts of this volume; in this section we provide a brief description of some of the predictions.

In the recent model proposed by Arkani-Hamed, Dimopoulos, and Dvali (ADD), unification can occur at a scale $L^{-1}=M^{*}<<M_{P}$, with the relative weakness of the gravitational interaction arising as a consequence of $n$ "large" extra dimensions in which gravitons propagate but the Standard Model fields do not [3]. The size $R$ of the extra dimensions is related to the unification scale and the usual Planck scale by:

$$
R^{n}=M_{P}^{2} / M^{*(2+n)}
$$




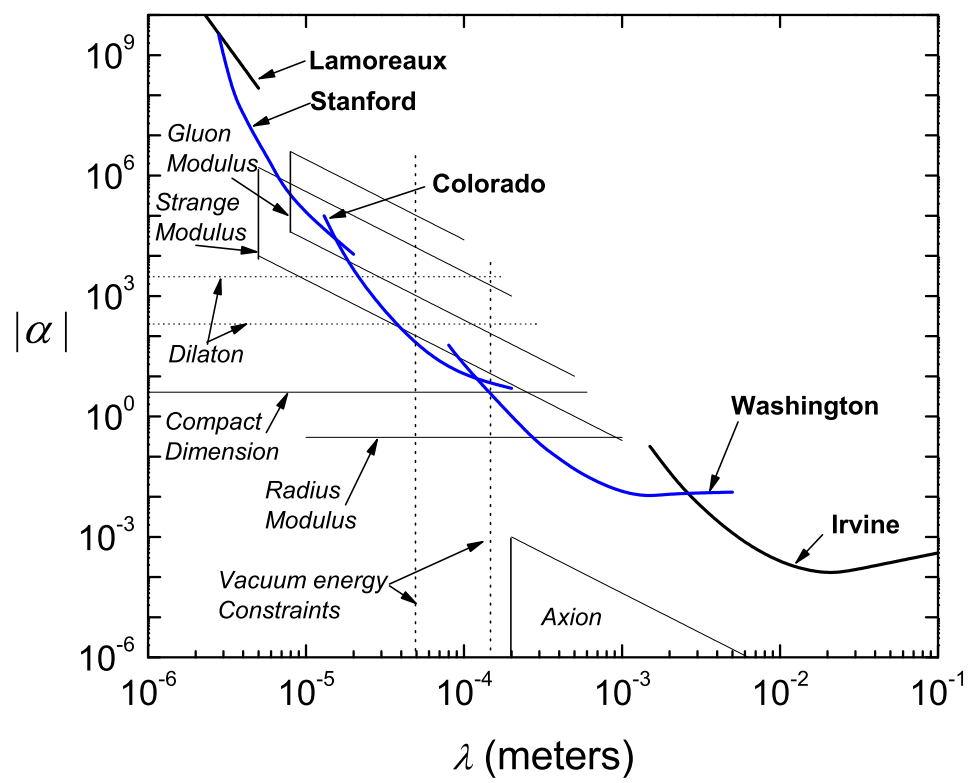

FIG. 1: Parameter space for deviations from Newtonian gravity in which the strength $\alpha$ of a hypothetical new Yukawa-type force is plotted versus the range $\lambda$. The current experimentally excluded region is above and to the right of the solid, bold curves. Curves in black are published results; curves in blue are unpublished but available in preprints. (A slightly weaker limit from the Washington group has been published 22].) Curves labeled Stanford and Irvine are $1 \sigma$ limits; others are $2 \sigma$ limits. Theoretical predictions are represented by the fine and dashed lines. For the moduli, dilaton, and compact dimension theories, the upper bounds on $\lambda$ of the regions shown are set at the approximate experimental limits known at the time the theories were proposed; the same is true of the $\alpha$ upper bounds for the vacuum energy prediction.

Note: As this work was being submitted, the authors were informed of the publication of the Colorado curve above [34].

Setting $M^{*}=1 \mathrm{TeV}$, and effectively eliminating the hierarchy problem (the vast discrepancy or "desert" between the weak and Planck scales), the choice of $n=1$ implies $R \sim 10^{13} \mathrm{~m}$, clearly ruled out by astrophysics. However, the choice of $n=2$ implies $R \sim 1 \mathrm{~mm}$, with the consequence that the gravitational force will behave according to a $1 / r^{4}$ law below this scale. As the scale $R$ is approached from above, Yukawa corrections are predicted; the model illustrated in Fig. 1 predicts $\alpha=4$ [4, 5]. In the absence of signals for these large compact dimensions, short-range gravity experiments can set limits on the unification scale 
$M^{*}$, which is also probed directly in collider experiments.

A consequence of eliminating the hierarchy problem in favor of embedding the Standard Model on a 3-dimensional brane in a higher dimensional bulk is that the success of the "desert" in explaining the approximate symmetries responsible for a variety of phenomena (e.g., small neutrino masses and proton stability) no longer applies. In response to these issues, two authors of the ADD model consider a scenario in which the appropriate symmetry-breaking processes occur at a higher order on distant branes, with suppressed information about this breaking transmitted to the Standard Model brane via messenger fields [7]. The messenger fields should be easily detectable by a short-range macroscopic force search if they have mass of $10^{-3} \mathrm{eV}$ or less, as they are predicted to have couplings on the order of $\alpha \sim 10^{6}$.

Several predictions arise in supersymmetric models with extra dimensions compactified on the order of the weak scale. Antoniadis, Dimopoulos and Dvali consider a model in which supersymmetry is broken at $\sim 1 \mathrm{TeV}$ by a compactification process, and show that the modulus associated with the dimension left at this scale acquires mass in the sub-eV range and a coupling of $\alpha \sim 1 / 3[9]$. The prediction for this "radius" modulus is shown in Fig. 1. Considering the problem of the stabilization of the extra dimensions in related models, Chacko and Perazzi predict a scalar field or "radion" corresponding to the size fluctuations of $\mathrm{TeV}$-sized extra dimensions with a coupling of $\alpha \sim 30$ and a range $\lambda \sim 40 \mu \mathrm{m}[\underline{6}]$.

The other moduli predictions in Fig. 1 derive from an earlier model in which supersymmetry is broken near $100 \mathrm{TeV}$ by a gauge-mediated process. This leads to moduli with masses in the sub-eV range and couplings as strong as $\alpha \sim 10^{6}[$ [ ] $]$.

The dilaton is another scalar in string theory whose vacuum expectation value determines the string coupling constant. Dilaton mass can also arise from supersymmetry breaking but is generally sensitive to unknown physics. Predictions for the dilaton coupling vary widely in the literature; the lower and upper bounds in Fig. 1 are representative 10, 11, 12].

Fig. 1]also shows two predictions of new macroscopic effects not directly related to string theory. The axion, a light pseudoscalar motivated by the strong $\mathrm{CP}$ problem of $\mathrm{QCD}$, is predicted to mediate forces between unpolarized test masses [13]. The allowed range between $200 \mu \mathrm{m}$ and $20 \mathrm{~cm}$ in Fig. [1 1 is constrained by laboratory and astrophysical observations [14], and the coupling by measurements of the neutron electric dipole moment. The remaining prediction is motivated by the cosmological constant $(\Lambda)$ problem, the discrepancy between 
the observed flatness of the universe and the extreme curvature expected from the vacuum energy contributions of the Standard Model fields. Several models [15, 16, 17] have attempted to address this problem by introducing new interacting quanta with a range on the order of $\Lambda^{-1 / 4} \sim 100 \mu \mathrm{m}$.

\section{EXPERIMENTAL CHALLENGES}

The principal limitation to testing gravity at short distances is the scaling of the signal force with the size of the apparatus. If all dimensions of a gravitational experiment are scaled by the same factor $r$, gravitational attraction behaves as $r^{4}$, so that signal forces become extremely weak at small distances. For example, expressing the gravitational force between two spherical test objects (radius $\sim$ separation $\sim r$ ) in terms of their densities $\rho$ yields: $F \sim G \rho_{1} \rho_{2} r^{4}$. For typical test mass densities $\left(\rho \sim 20 \mathrm{~g} / \mathrm{cm}^{3}\right)$, substituting $r=10 \mathrm{~cm}$ gives $F \sim 10^{-5} \mathrm{~N}$. Measuring a force of this magnitude was the challenge met by Cavendish with his torsion balance experiment, published in 1798. In order to measure gravity at $100 \mu \mathrm{m}$, the experimenter is faced with scaling the size of the test masses to this range as well, so as not to be overwhelmed by the attraction of the additional mass at larger scales. Setting $r=100 \mu \mathrm{m}$ yields $F \sim 10^{-17} \mathrm{~N}$, which explains in large part why it has taken two centuries to achieve measurements sensitive to gravitational effects below a millimeter.

At the same time, backgrounds due to known physics increase rapidly at short distances.

Electrostatic effects due to surface potentials increase as $r^{-2}$, and magnetic forces due to contaminants increase as $r^{-4}$. At the lower end of the range considered $(\sim 1 \mu \mathrm{m})$, the Casimir force (of experimental interest itself) also becomes visible and quickly begins to dominate. The following sections are devoted to a description of current short-range experiments, with emphasis on the strategies used to overcome the dual challenge of extremely small signals and rapidly increasing backgrounds.

\section{CLASSICAL GRAVITATION EXPERIMENTS}

The Irvine 2-5 cm Experiment and Cryogenic Torsion Pendulum

The highly linear and extremely sensitive torque response of thin filaments under tension has made the torsion balance the instrument of choice for measuring weak forces at labo- 
ratory scales. Between $3 \mathrm{~mm}$ and $3 \mathrm{~cm}$, the best constraints on new forces derive from the 2-5 cm experiment in the group of R. Newman at the University of California at Irvine [18]. In this experiment, a cm-sized cylindrical test mass was suspended from one end of a torsion balance inside a long massive tube (a null geometry for $1 / r^{2}$ forces). As the tube was translated periodically perpendicular to its axis, no deflection of the balance above that associated with a small Newtonian edge effect was observed. Torque sensitivities of about $10^{-14} \mathrm{Nm}$ were obtained, yielding the limit curve in Fig. 11.

A principal limitation to the sensitivity of this experiment was the effect of tilt. Motion of the tube along its guide track caused a slight tilt in the large block supporting the balance and a resulting phase-correlated motion of the balance suspension point. While the tilt was largely compensated with external instrumentation, the residual effect remained one of the main sources of systematic error. Magnetic effects presented another large source of systematic uncertainty, while torque measurement sensitivity was limited by a combination of instrument noise, seismic noise, and spurious gravitational forces not associated with the motion of the tube.

For the past several years, the Irvine group has been constructing a torsion pendulum which operates at cryogenic temperatures $(T \sim 2 \mathrm{~K})$, in collaboration with the group of P. Boynton at the University of Washington (Fig. 2). This approach has several advantages [19], of which we mention only a few. For one, the pendulum may be operated in the frequency mode, which is highly insensitive to the effect of tilt. In this method, pioneered by the Boynton group [20], the resonant frequency of the pendulum is monitored for the shifts induced by the force gradient generated by an external source mass. At room temperature, the temperature dependence of the torsion fiber elastic properties limits the usefulness of this mode, but at cryogenic temperatures this dependence is small and temperature stability is easy to maintain. Operation at cryogenic temperature also allows for the use of superconducting shielding to reduce magnetic backgrounds.

Finally, cryogenic operation can reduce instrument noise considerably, inasmuch as this effect is dominated by thermal noise due to dissipation in the force-sensitive detector. In the thermal noise limit, the sensitivity of this experiment (and all others discussed below) to new forces is given by:

$$
\alpha \sim \frac{1}{\rho_{1} \rho_{2}} \sqrt{\frac{\omega T}{Q \tau}}
$$




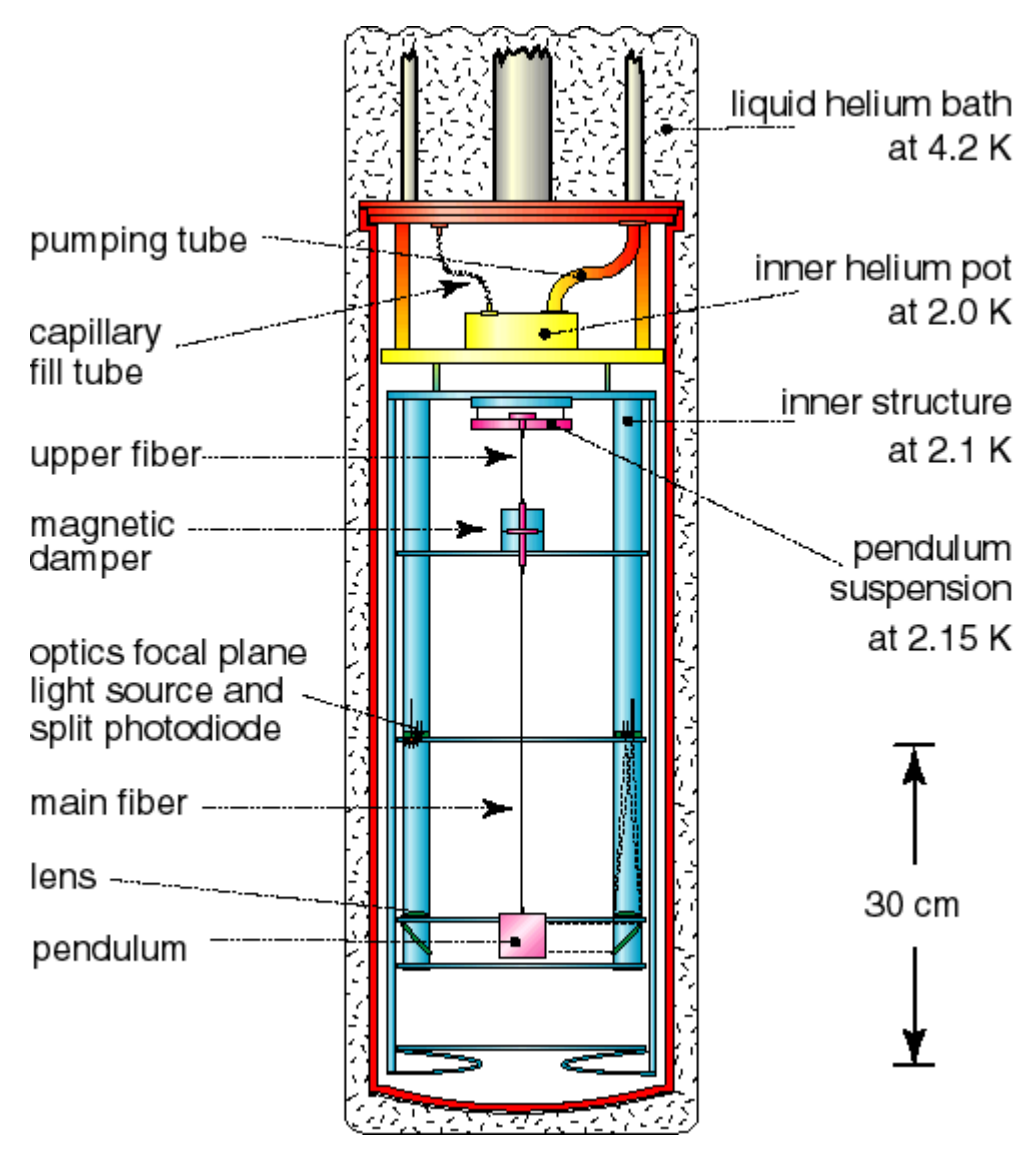

FIG. 2: Schematic of the Irvine-Washington cryogenic torsion pendulum. Source masses are suspended alongside the cryostat at the level of the pendulum mass. Source: http://www.physics.uci.edu/gravity/Gcryo.htm

where $\rho_{1}$ and $\rho_{2}$ are the test mass densities, $\omega$ is the operational frequency of the experiment, $T$ is the temperature, $Q$ is the detector mechanical quality factor, and $\tau$ is the experimental integration time. In addition to the improvement in sensitivity expected from a reduction in $T$ from $300 \mathrm{~K}$ to $2 \mathrm{~K}$, further improvement can be expected from a gain in $Q$ which is observed in many materials at low temperatures.

Construction of the cryogenic experiment is complete and a precision measurement of the Newtonian constant $G$ is in progress. A test of the inverse square law is planned which will have maximum sensitivity for forces with a range of about $15 \mathrm{~cm}$. For the latter experiment, additional sensitivity is expected from a re-optimized test mass design based on a harmonic expansion of the mass and field multipole moments. In a method similar to that used in previous equivalence principle tests, the pendulum and source mass are configured to 
suppress Newtonian mass and field moments, respectively, so that classical gravitational effects enter into the measurement only to second order or higher [21]. At the same time, non-Newtonian moments can be enhanced. If all noise sources can be reduced to near the level of the thermal noise, an improvement in sensitivity of about two orders of magnitude over previous results is expected, corresponding to the projected limit shown in Fig. 8 ,

\section{The Eöt-Wash Short-Range Experiment}

Between $100 \mu \mathrm{m}$ and $3 \mathrm{~mm}$, the most sensitive limit on new forces has been attained by the short-range torsion pendulum experiment in the Eöt-Wash group at the University of Washington (Fig. [3) 22]. The force-sensitive part of the torsion pendulum consists of a $1 \mathrm{~mm}$ thick aluminum annulus with an array of ten equally spaced holes. The source mass consists of a stack of two copper disks, each a few millimeters thick, with similar arrays of holes. The source rotates approximately once every two hours, torquing the pendulum 10 times per revolution. This arrangement has several clever features. For one, the signal occurs at 10 times the revolution rate, allowing easy discrimination from vibrations associated with the source drive. Also, in the source stack, the angular position of the lower disk is offset relative to the upper disk so as to cancel the torque associated with the long-range Newtonian attraction between the test masses, while leaving possible sub-millimeter forces unaffected.

Not shown in Fig. 3, an essential component of the experiment is an electrostatic shield. This is made from a $20 \mu \mathrm{m}$ thick beryllium-copper foil stretched on a frame between the source and pendulum.

The torque as a function of test mass separation was measured for two prototypes of this apparatus. Torque sensitivities of about $10^{-16} \mathrm{Nm}$ were attained, and no deviation from the expected Newtonian signal was observed for test mass separations down to about $200 \mu \mathrm{m}$. From these data, the preliminary results shown in Fig. 1 were obtained [23]. This is the shortest-ranged experiment to date to have attained gravitational sensitivity.

Improvements to this experiment currently under way include thinner pendulum and source disks, both constructed of copper or molybdenum for greater test mass density. The hole arrangements have been further optimized for Yukawa signals and cancellation of Newtonian gravity. Better vibration isolation and a thinner shield should significantly 


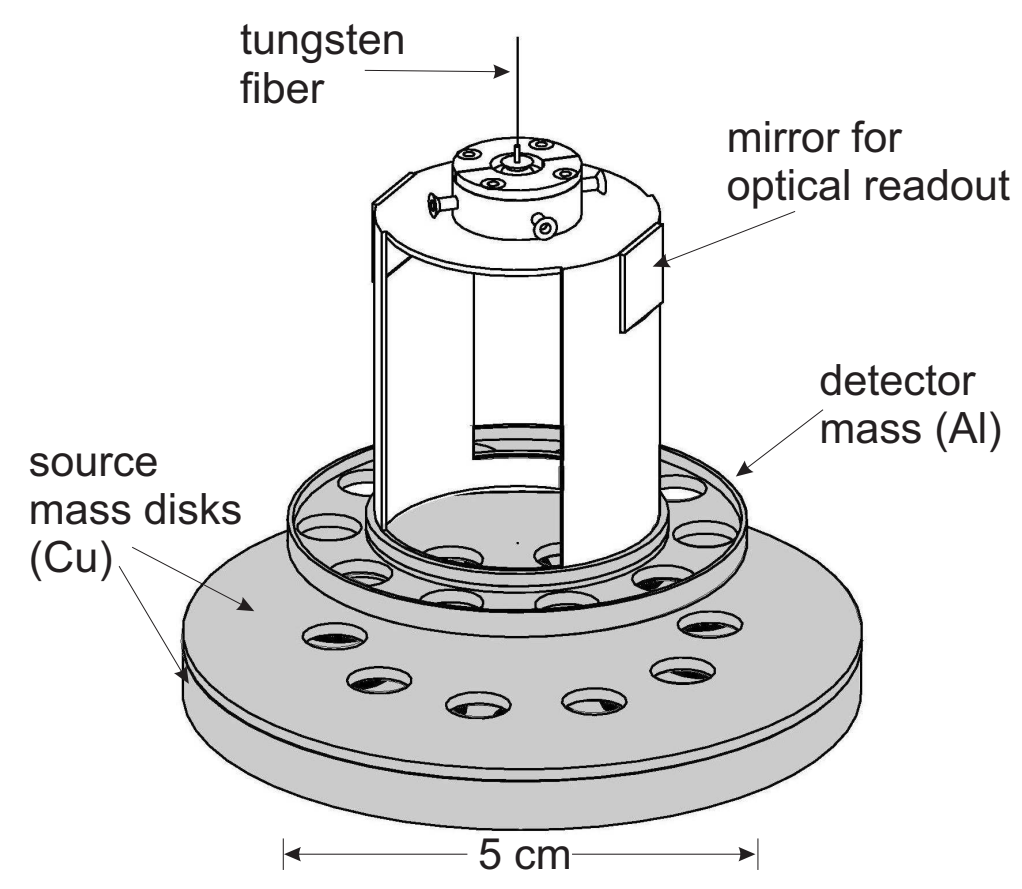

FIG. 3: Eöt-Wash short-range torsion pendulum experiment. Source: Ref. 22]

reduce the test mass separation. The projected limits expected with this technique are shown in Fig. 8

\section{HIGH FREQUENCY TECHNIQUES}

With low operating frequencies and high mechanical quality factors $\left(\sim 10^{5}\right)$, torsion balance experiments are very attractive from the point of view of thermal noise, even at room temperature. However, their sensitivities are usually limited by other backgrounds, including low-frequency vibrations which can also limit the minimum practical test mass separation. Recently, several experiments have been developed using high-frequency techniques, which show promise for operation at the thermal noise limit and for attaining smaller test mass separation.

\section{Colorado Torsional Oscillator Experiment}

An approach for improving the experimental sensitivity at $100 \mu \mathrm{m}$ and below has been pursued for the past several years by the authors and their collaborators at the University 


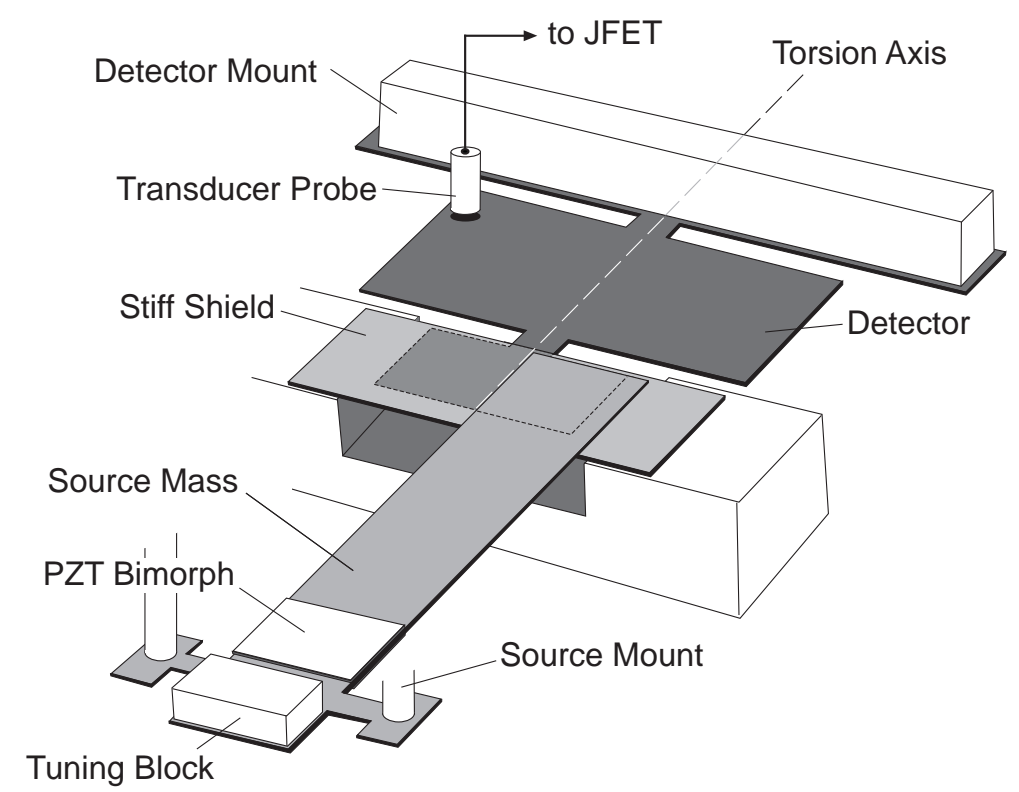

FIG. 4: Central components of Colorado experiment. Figure is to scale. The source mass has approximate dimensions $7 \mathrm{~mm} \times 40 \mathrm{~mm} \times 0.25 \mathrm{~mm}$ and is driven at the detector resonance by a PZT (lead zirconate titanate) bimorph. Detector motion is monitored with a capacitive probe suspended above a rear corner and connected to a JFET amplifier.

of Colorado [24, 25]. The basic design is shown in Fig. 4. Planar test mass geometry is used in order to concentrate as much of the available test mass density as possible at the range of interest. The flat plates are nominally a null geometry with respect to $1 / r^{2}$ forces, which is important for suppressing Newtonian forces relative to new short-range effects. The test masses are fabricated from tungsten wafers, approximately 250 microns thick. The source mass consists of a $4 \mathrm{~cm}$ long cantilever, which is driven mechanically at a natural resonant frequency of the detector mass, nominally $1 \mathrm{kHz}$. The detector consists of two coplanar rectangles joined along their central axes by a short segment. In the resonant mode of interest, the detector rectangles counter-rotate in a torsional mode about the axis defined by the segment.

Electrostatic and acoustic backgrounds are suppressed with a stiff conducting shield, consisting of a gold-plated sapphire wafer, between the test masses. The shield currently limits the minimum test mass separation to approximately 100 microns.

Operation of the experiment at the detector resonant frequency, while increasing the experimental sensitivity, places strict requirements on vibration isolation. At the operational 
frequency of $1 \mathrm{kHz}$ it is possible to construct a simple passive vibration isolation system, in which the test masses and shield are each suspended from a series of brass disks connected by fine wires under tension. This system provides about $200 \mathrm{~dB}$ of attenuation at the operational frequency [26]. Modular design, visibility, and easy control of test mass positioning and electrical connections allow for rapid characterization of observed signals in situ.

Electromagnetic, acoustic and vibrational backgrounds have been sufficiently suppressed such that the experiment is currently limited by detector thermal noise. To reduce the thermal background, the detector is annealed at $1300 \mathrm{C}$ for several hours before installation in the experiment, which significantly increases its mechanical quality factor.

In a recent series of runs, no signal above thermal noise was observed after 22 hours of integration time. This corresponded to a force sensitivity of about $10^{-15} \mathrm{~N}$, from which the limit derived in Fig. 1] is obtained [27]. Development of a stretched-membrane shield and flatter test masses with higher $\mathrm{Q}$ is in progress. In the absence of backgrounds, the limits attainable with the current sensitivity of the experiment (at $300 \mathrm{~K}$ ) at half the present minimum test mass separation are shown in Fig. 8] A cryogenic version of this experiment, with a $4 \mathrm{~K}$ operating temperature and an expected detector $Q$ enhancement of an order of magnitude, could improve the sensitivity by an additional factor of 40 .

\section{Stanford Micro-cantilever Experiment}

Another high-frequency measurement is in progress in the group of A. Kapitulnik at Stanford. This experiment uses techniques of scanning force microscopy and microfabrication to probe distance scales on the order of a few tens of microns (Fig. 51). The force-sensitive detector mass consists of a $50 \mu \mathrm{m}$ gold cube mounted on the end of a silicon nitride cantilever $250 \mu \mathrm{m}$ long and less than $1 \mu \mathrm{m}$ thick.

The source mass consists of a parallel array of alternating strips of gold and silicon, each $100 \mu \mathrm{m}$ wide. It is scanned in the horizontal plane below the detector in the direction perpendicular to the strips, with the resulting periodic variations in density providing the driving mass-coupled force. This design offers an advantage similar to that of the Eöt-Wash torsion pendulum, in that the frequency of the source drive mechanism can be set much lower than that of the expected signal. In the Stanford experiment, the mass modulation frequency is matched to the detector cantilever resonance (about $300 \mathrm{~Hz}$ ), resulting in less 


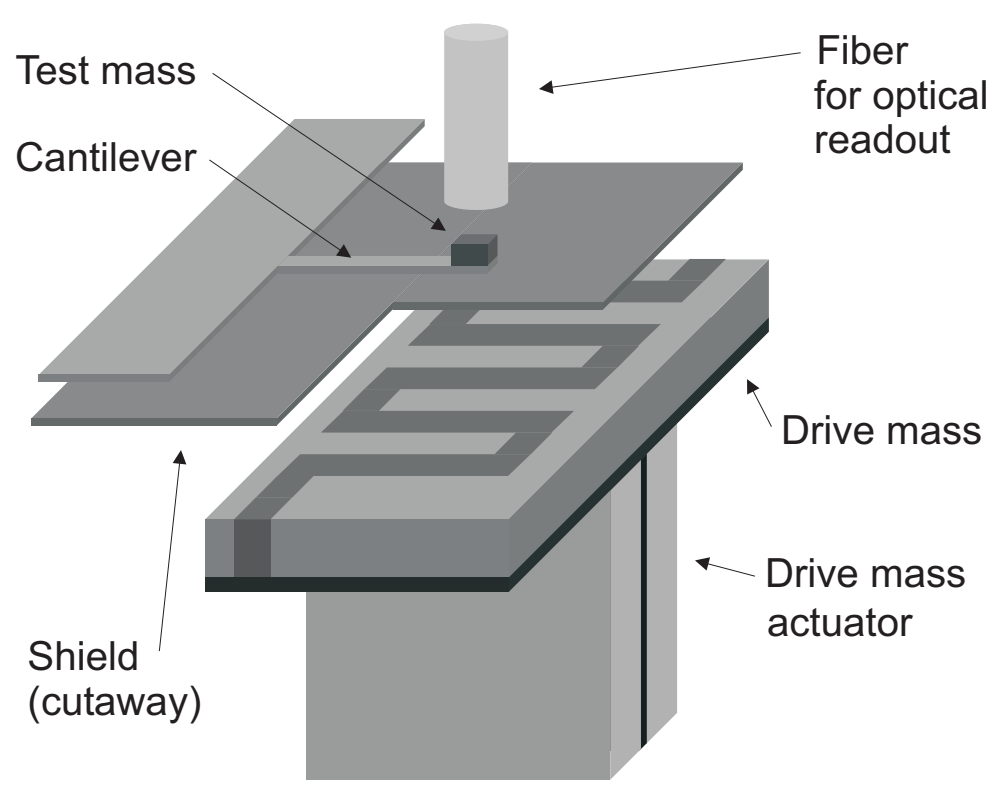

FIG. 5: Central components of the micro-cantilever experiment at Stanford. Figure not to scale; test mass in actual apparatus is approximately a $50 \mu \mathrm{m}$ cube suspended $15 \mu \mathrm{m}$ above shield. Source: Chiaverini J., "Small Force Detection Using Microcantilevers: Search for Sub-millimeter Range Deviation from Newtonian Gravity," Doctoral Thesis, Stanford University, 2002 (unpublished).

of a burden on vibration isolation than for gap-modulated experiments.

An electrostatic shield, fabricated from a silicon nitride membrane $3 \mu \mathrm{m}$ thick with a $100 \mathrm{~nm}$ gold plating, is placed between the test masses. It is attached to the base of the cantilever mount, leaving a gap of $15 \mu \mathrm{m}$ below the detector mass.

The limiting background to this measurement is expected to be thermal noise of the force-sensitive cantilever. To reduce this background, the experiment is inserted into a liquid helium dewar for operation at $T \sim 10 \mathrm{~K}$, where the expected force sensitivity is about $10^{-17} \mathrm{~N}$ for integration times of a few hours.

After 20 minutes of integration time during a run in 2002, data from this experiment showed evidence of a signal approximately three times greater than the expected thermal noise [28]. The signal appeared most likely to be an electrostatic background, due to modulation of the shield caused by surface variations on the source, and a $0.3 \mathrm{~V}$ potential between the shield and the detector mass. The phase and magnitude of the observed signal was not consistent with a mass-coupled force generated by the source mass, and the data were used to obtain the preliminary limits shown in Fig. 1 representing an upper limit on such forces. 


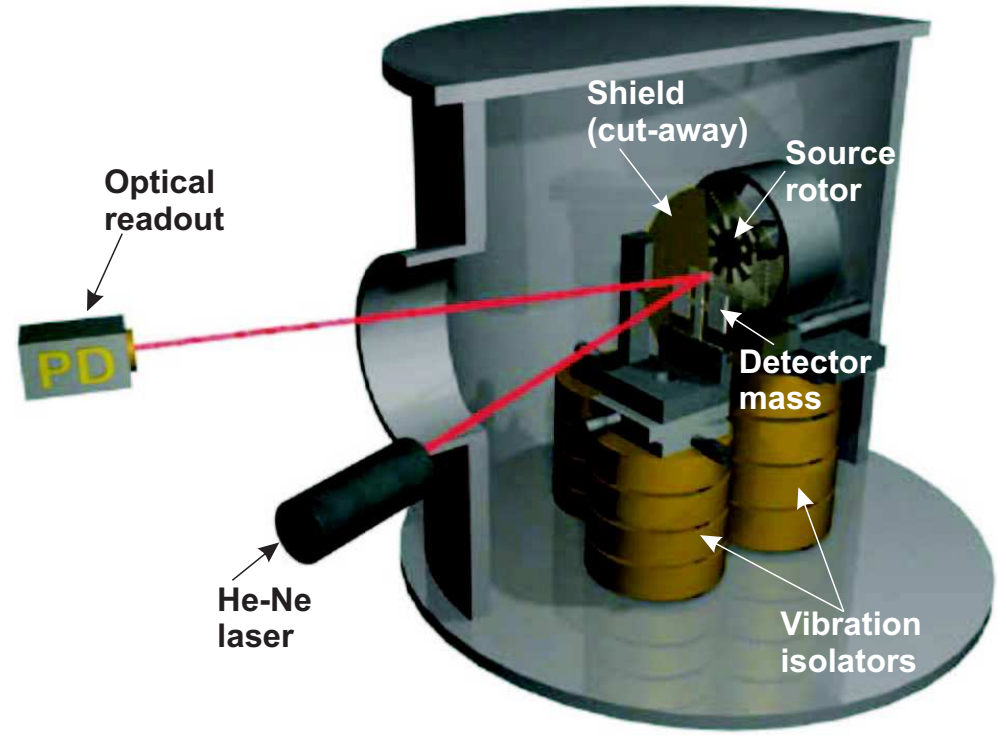

FIG. 6: Experiment at Dusseldorf: cut-away view of vacuum chamber. Source: Haiberger L., Lümmen N., Schiller S., "A Resonant Sensor for the Search for Deviations from Newtonian Gravity at Small Distances," May 2001 (unpublished), also available at http://www.exphy.uni-duesseldorf.de/ResearchInst/WelcomeFP.html

While a projected limit was not available as of this writing, improvements to the experiment continue, including a new source mass with additional conducting and insulating layers above the strips to eliminate surface variations.

\section{Dusseldorf Torsional Oscillator Experiment}

An experiment in progress in the group of S. Schiller at the University of Dusseldorf introduces several new features. An early conception is illustrated in Fig. 6. Planar geometry is used, with both test masses mounted vertically. The detector mass, fabricated from a high-purity silicon wafer, is a torsional oscillator similar in design to the Colorado detector. Excitation of the source mass at the detector resonance (about $5 \mathrm{kHz}$ ) is accomplished using a rotating disk with nine tungsten alloy teeth embedded in a lower-density substrate.

A thin metal plate is placed between the test masses as an electrostatic shield. All elements are mounted atop multi-stage vibration isolation stacks, consisting of viton rings sandwiched between $1 \mathrm{~kg}-$ mass brass disks. 
The sensitivity of this experiment is also expected to be limited by thermal noise. In the thermal-noise limit, the reduction in sensitivity associated with the use of a low-density silicon detector is offset somewhat by the exceptionally high mechanical quality factors attainable, which are about an order of magnitude larger than those observed so far in metal oscillators at room temperature. Plans for operation of the experiment at $4 \mathrm{~K}$ are under way, where improvement in $Q$ of at least two additional orders of magnitude can be expected for silicon detectors. The combined effect of the increase in $Q$ with the reduction in temperature is reflected in the very sensitive projected limits for this experiment, shown in Fig. 8

A room-temperature prototype of the experiment has been assembled and is under test. In early runs, a signal approximately 10 times larger than the detector thermal noise was observed. As the signal was present even for large separations of the test masses, a vibrational background was suspected. This background has been reduced with several improvements in the design, including a more uniform source disk and remote positioning of the drive vibration isolation stack. A cryostat for the $4 \mathrm{~K}$ version of the experiment is also under construction.

\section{Padova Casimir Force Experiment}

Below test mass separations of a few microns, the principal background to macroscopic force searches is expected to be the Casimir effect, a force which arises due to zero-point fluctuations of the electromagnetic field. At the moment, the best limits on new forces in this range are derived from direct measurements of this effect, in general by ascertaining the maximum possible size of a Yukawa-type force for the Casimir plates still consistent with the experimental residuals. Below $\lambda=3 \mu \mathrm{m}$, the best published limits are obtained from the measurement by Lamoreaux in 1997 [2, 24] which used a torsion balance to measure the Casimir force between a flat gold-plated disk and a thin spherical shell [29].

An experiment at Legnaro laboratories by a joint team of the INFN, Padova and Pavia, and the University of Padova, uses a frequency-shift technique to measure the Casimir force between parallel conducting plates (Fig. (7) [30]. The force-sensitive detector plate, a $47 \mu \mathrm{m}$

thick, $2 \mathrm{~cm}$ long silicon reed with a $50 \mathrm{~nm}$ chromium plating, is clamped at its base and driven electrically into oscillation at its lowest natural frequency of about $138 \mathrm{~Hz}$. The source 

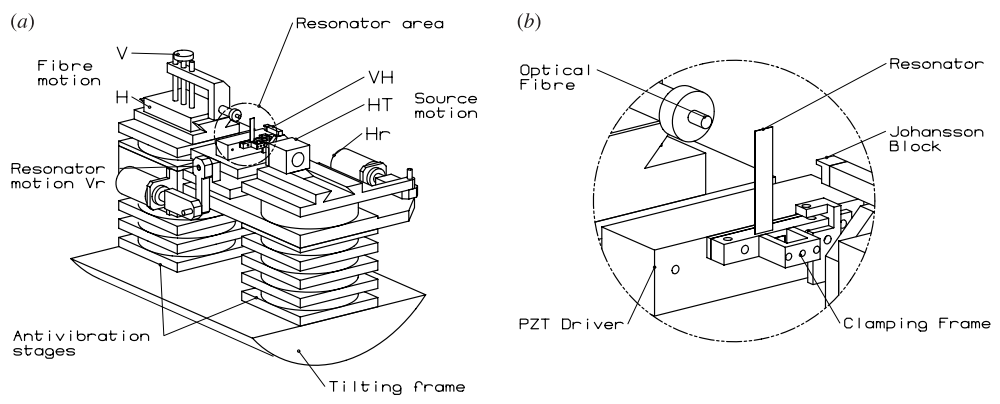

FIG. 7: (a) Central components and (b) detail of the Casimir force experiment at Legnaro laboratories. The source mass is mounted on the Johansson block. Source: Ref. 32].

mass, a $5 \mathrm{~mm}$ thick, chromium-plated silicon block, is mounted on a nearby translation stage and brought into very close proximity to the detector. The detector resonance is monitored optically for the frequency shifts induced by static external force gradients.

The apparatus is mounted on passive vibration isolation stacks, and in turn into a large scanning electron microscope (not shown in Fig. 7) in order to monitor the cleanliness and parallelism of the test mass surfaces at the micron-sized separations needed for this measurement. The electrostatic background is due primarily to contact potentials in the circuit connecting the test masses. It is eliminated to first order by nulling the static force between the test masses at small distances, and the remaining background is subtracted off after careful fits to the measured force in the presence of applied potentials at larger gaps.

This experiment was able to obtain a measurement of the Casimir force to a precision of $15 \%$, the first unambiguous measurement of this effect for the parallel-plate geometry. An earlier version of this experiment was among the first high-frequency measurements used to derive limits on new effects [31]. While those limits were not competitive with the most stringent results at that time, a re-optimized version of the present apparatus is expected to improve the limits by at least an order of magnitude in the range near $1 \mu \mathrm{m}$, as shown in Fig. 8] 32].

\section{CONCLUSION}

Motivated in large part by suggestions of extra dimensions, three of the experiments described above have explored approximately 7 square decades of the parameter space for 


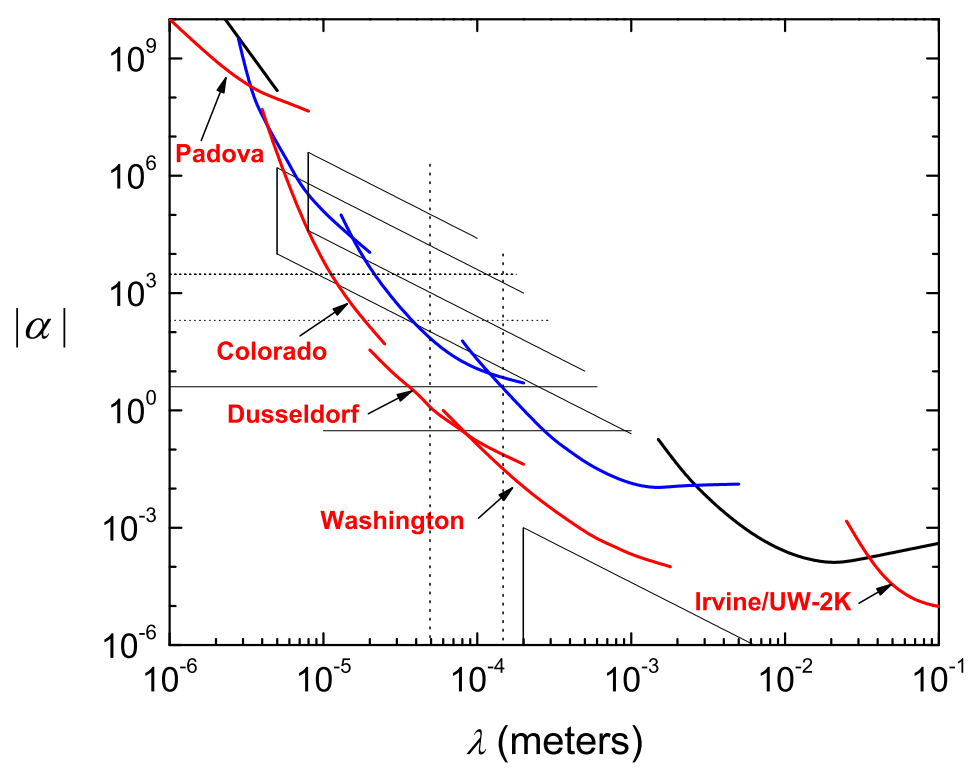

FIG. 8: The projected limits for several experiments currently in progress (red). The Irvine-UW curve is from M. Moore (private communication). The Washington curve is from http://www.npl.washington.edu/eotwash/shortr.html, and the Dusseldorf curve from Haiberger L., Lümmen N., Schiller S., "A Resonant Sensor for the Search for Deviations from Newtonian Gravity at Small Distances," May 2001 (unpublished).

new forces in the past five years [33], and have set significant limits on new physics. The maximum possible size of any extra dimension in the ADD scenario has been limited to $200 \mu \mathrm{m}$ (or $150 \mu \mathrm{m}$ for $n=2$ equal-sized extra dimensions, corresponding to a lower limit on the unification scale of $M^{*} \geq 4 \mathrm{TeV}$ ) [23], a new upper limit on the dilaton mass has been set at $8.6 \times 10^{-3} \mathrm{eV}$ (assuming a limit on the coupling of $\alpha<2000$ ), and much of the remaining parameter space for the moduli force scenario of Ref. 8] has been excluded.

While the torsion pendulum remains the instrument of choice for scales above 100 microns, high-frequency experiments show great potential for improving the limits at shorter ranges. Fig. 8 shows the projected limits for the next few years, considering only some of the experiments currently in progress. Experimental coverage of at least 7 additional square decades of parameter space, with investigation of the region of cosmological interest at gravitational strength, seems well within reach in the near future. 


\section{Acknowledgements}

We wish to thank R. Newman, P. Boynton, and M. Moore for description of the cryogenic torsion pendulum experiment, L. Haiberger and S. Schiller for materials describing the Dusseldorf experiment, and E. Adelberger, J. Chiaverini, C. D. Hoyle, A. Kapitulnik, R. Onofrio, and G. Ruoso for useful materials and comments.

[1] Fischbach E., Krause D. E., Mostepanenko V. M., Novello M., Phys. Rev. D 64 (2001) 07501.

[2] Bordag M., Mohideen U., Mostepanenko V. M., Phys. Rept. 353 (2001) 1.

[3] Arkani-Hamed N., Dimopoulos S., Dvali G., Phys. Lett. B 429 (1998) 263.

[4] Floratos E. G., Leontaris G. K., Phys. Lett. B 465 (1999) 95.

[5] Kehagias A., Sfetsos K., Phys. Lett. B 472 (2000) 39.

[6] Chacko Z., Perazzi E., arXiv:hep-ph/0210254.

[7] Arkani-Hamed N., Dimopoulos S., Phys. Rev. D 65 (2002) 052003.

[8] Dimopoulos S., Giudice G., Phys. Lett. B 379 (1996) 105.

[9] Antoniadis I., Dimopoulos S., Dvali G., Nucl. Phys. B 516 (1998) 70.

[10] Taylor T. R., Veneziano G., Phys. Lett. B 213 (1988) 450.

[11] Ellis J., Kalara S., Olive K. A., Wetterich C., Phys. Lett. B 228 (1989) 264.

[12] Kaplan D. B., Wise M. B., J. High Energy Phys. 0008 (2000) 037.

[13] Moody J. E., Wilczek F., Phys. Rev. D 30 (1984) 130.

[14] Rosenberg L. J., van Bibber K. A., Phys. Rep. 325 (2000) 1.

[15] Sundrum R., J. High Energy Phys. 7 (1999) 1.

[16] Beane S. R., Gen. Rel. Grav. 29 (1997) 945.

[17] Schmidhuber C., arXiv:hep-th/0207203.

[18] Hoskins J. H., Newman R. D., Spero R., Schultz J., Phys. Rev. D 32 (1985) 3084.

[19] Newman R., Class. Quantum Grav. 18 (2001) 2407.

[20] Boynton P. E., Class. Quantum Grav. 17 (2000) 2319.

[21] Moore M. W., Bourdreaux A., DePue M., Guthrie J., Legere R., Yan A., Boynton P. E., Class. Quantum Grav. 11 (1994) A97.

[22] Hoyle C. D., Schmidt U., Heckel B. R., Adelberger E. G., Gundlach J. H., Kapner D. J., 
Swanson H. E., Phys. Rev. Lett. 86 (2001) 1418.

[23] Adelberger E. G., for the Eöt-Wash collaboration, arXiv:hep-ex/0202008

[24] Long J. C., Chan H. W., Price, J. C., Nucl. Phys. B 539 (1999) 23.

[25] Long J. C., Churnside A. B., Price, J. C., in: Gurzadyan V. G., Jantzen R. T., Ruffini R. (Eds.), Proceedings of the Ninth Marcel Grossmann Meeting on General Relativity, World Scientific, Singapore, 2002, part B, p. 1819.

[26] Chan H. W., Long J. C., Price J. C., Rev. Sci. Instrum. 70 (1999) 2742.

[27] Long J. C., Chan H. W., Churnside A. B., Gulbis E. A., Varney M. C. M., Price J. C., arXiv:hep-ph/0210004.

[28] Chiaverini J., Smullin S. J., Geraci A. A., Weld D. M., Kapitulnik A., arXiv:hep-ph/0209325.

[29] Lamoreaux S. K., Phys. Rev. Lett. 78 (1997) 5.

[30] Bressi G., Carugno G., Onofrio R., Ruoso G., Phys. Rev. Lett. 88 (2002) 041804.

[31] Carugno G., Fontana Z., Onofrio R., Rizzo C., Phys. Rev. D 55 (1997) 6591.

[32] Bressi G., Carugno G., Galvani A., Onofrio R., Ruoso G., Veronese F., Class. Quantum Grav. 18 (2001) 3943.

[33] For a review of the experimental limits in 1997, see Ref. [24].

[34] Long J. C., Chan H. W., Churnside A. B., Gulbis E. A., Varney M. C. M., Price J. C., Nature $421(2003) 922$. 